\title{
Monte Carlo Convex Hull Model for Classification of Traditional Chinese Paintings
}

\author{
Meijun Sun ${ }^{\mathrm{a}}$, Dong Zhang ${ }^{\mathrm{a}}$, Zheng Wang ${ }^{\mathrm{b}}$, Jinchang Ren ${ }^{\mathrm{c}}$, Jesse S. Jin ${ }^{\mathrm{b}}$ \\ ${ }^{a}$ Media Technology \& Systems Research Lab, School of Computer Science\& Technology, Tianjin University, 300072 Tianjin, \\ China \\ ${ }^{b}$ School of Computer Software, Tianjin University, 300072 Tianjin, China \\ ${ }^{c}$ Centre for excellence in Signal and Image Processing, University of Strathclyde, Glasgow, U.K
}

\begin{abstract}
While artists demonstrate their individual styles through paintings and drawings, how to describe such artistic styles well selected visual features towards computerized analysis of the arts remains to be a challenging research problem. In this paper, we propose an integrated feature-based artistic descriptor with Monte Carlo Convex Hull $(\mathrm{MCCH})$ feature selection model and support vector machine (SVM) for characterizing the traditional Chinese paintings and validate its effectiveness via automated classification of Chinese paintings authored by well-known Chinese artists. The integrated artistic style descriptor essentially contains a number of visual features including a novel feature of painting composition and object feature, each of which describes one element of the artistic style. In order to ensure an integrated discriminating power and certain level of adaptability to the variety of artistic styles among different artists, we introduce a novel feature selection method to process the correlations and the synergy across all elements inside the integrated feature and hence complete the proposed style-based descriptor design. Experiments on classification of Chinese paintings via a parallel MCCH model illustrate that the proposed descriptor outperforms the existing representative technique in terms of precision and recall rates.
\end{abstract}

Keywords: Monte Carlo, Artistic style descriptor, Feature selection, Classification of Chinese paintings;

\section{Introduction}

With the popularity of World Wide Web and the well-advanced digital media and intelligent information processing technologies, fine art paintings have become an important part of media resources. Managing collections of digitized art pieces at a large scale, including their indexing and retrieval, becomes a challenge that needs to be addressed. As an important part of the world's cultural heritage, many invaluable antique Chinese paintings are digitized and exhibited on the Internet.

Recently, image classification aims at associating images with semantic labels automatically [1][2]. Typically, learning vocabulary by applying k-means clustering on image patches (also called bag-of-word) [3] has been proven very useful and effective for image classification. But it ignores the spatial information as well as correlations among local features. So, a lot of studies [4]-[11] have been done to overcome the problems. To reduce the training complexity and improve image classification performance, sparse coding based linear spatial pyramid matching methods [12]-[15] are proposed which help to improve classification performance.

However, Chinese paintings are distinguished from Western art in that it is purely executed with the Chinese brush, Chinese ink, mineral, and vegetable pigments. In particular, many Chinese artists used monochromic ink to paint and sometimes do not even possess gradually changing tones. Further, Chinese painting is often regarded as an integrated form of art with calligraphy, for which color is usually not used. As a result, Chinese paintings primarily rely on the use of brush strokes to represent the style of an artist. Therefore, arts work such as Chinese paintings need special image analysis methods. For instance, a significant style of Chinese painting is "Mountain-Water paintings", in which mountains, stones, trees, rivers, waterfalls and small pagodas are prominent in this art form.

While classification of traditional Chinese paintings shares the same problem with general image classification in principle, it also has certain level of uniqueness, which can be highlighted as: 1) 
Artistic styles of paintings are generic and subjective. 2) Objects in paintings can't be simply perceived in terms of real ones without further explanation and interpretations by artists. Recent work report starts to show the trend that a proper feature descriptor plays an important role in general image classification as well as paintings categorization [16] presented a mixture of two-dimensional multi-resolution hidden Markov model (MHMM) method to Black-and-white Chinese painting classification. However, the number of its parameters cannot be determined automatically, which could cause problems for applications that automatic classification is required [17] designed a system for the authenticity of paintings by using color and texture analysis technique [18] introduced an algorithm which tries to categorize traditional Chinese paintings into Gongbi (traditional Chinese realistic painting) and Xieyi (freehand style) via using low-level features and a SVM classifier. As Gongbi and Xieyi only represent a small part of Chinese painting skills, however, such techniques may have limited roles in describing the style of Chinese paintings [19] analyzed the choice of dimension, selection and weighting of visual words for Bow in image categorization. Due to the fact that Chinese paintings have the nature of nonrepresentational forms of painting styles, it is very difficult to extract the "words" for applying the reported techniques [20] surveyed the brush analysis and artist identification techniques [21] considered classification of classical western paintings in extracting local and global features via a RBF neural network based classifier. The performance is not competitive enough as the experimental results in terms of identification accuracy are reported to be below $70 \%$, indicating the level of difficulties in painting style descriptions [22] provided scene categorization via contextual visual words, and [23] reviewed the techniques to accelerate visual concept classification.

While significant efforts have been reported in the domains of digitized image categorization of Chinese paintings, little of them proposed a persuasive feature descriptor to characterize the artistic style embedded inside traditional Chinese paintings. In this paper, we propose an integrated art style descriptor via introducing a new concept of infusion factor to optimize the selection of individual elements for constructing the integrated art style descriptor. Therefore, in comparison with the existing approaches, our contribution of originality can be highlighted as: (i) propose an integrated approach to exploit the strength of individual features to characterize the artistic style; (ii) introduce a new concept of infusion factor to optimize the selection of individual feature elements and maximize the discriminating power of the integrated art descriptor; (iii) feature screening is made adaptive to different artistic styles to optimize the performance of automated classification of traditional Chinese paintings. A Monte Carlo Convex Hull Support Vector Machine (MCCH-SVM) model is proposed here, this model is consist of two parts including the MCCH based feature selection model and SVM classifier.

Experiments support that, through automated classification of traditional Chinese paintings, the proposed descriptor outperforms the existing representative technique in terms of precision and recall rates.

The rest of the paper is organized as follows. In Section 2, a range of local and global low-level features are discussed and analyzed in terms of their descriptive capabilities for different artistic styles in traditional Chinese paintings. Section 3 describes the proposed integrated artistic style descriptor through introduction of an infusion factor as well as the adaptive selection and examination scheme, based on which an automated classification algorithm is also described to validate the proposed artistic descriptor. Section 4 shows the comparison of experimental results, and finally, concluding remarks are drawn and reported in Section 5.

\section{Feature based analysis of Artistic Styles}

In the past decade, many effective real scenes image classification methods have been reported, such as Bag of Words, Spatial Pyramid Matching, Locality-constrained Linear Coding and Fisher Vector. These methods are mainly using local features of SIFT or HOG and they show good performance on real scenes images. However, under the effect of water and ink diffusion, most textures and edges in Chinese paintings are much smoother than real scenes images. This decreases the effectiveness of these local features in Chinese painting classification field. So in this section, we are introducing the specific feature descriptor for Chinese painting.

In traditional Chinese paintings, artistic styles are primarily represented and reflected in colors, | texture, composition and their strokes. Fig. 1 shows a set of sample paintings by two famous traditional Chinese artists, Qi Baishi (1864-1957) and Huang Binhong (1865-1955). As seen, the samples by Qi Baishi are simple, vivid and rich in flavor of life (top row). His artistic style is obviously different from 
that of Huang Binhong, an artist of modern China, whose paintings are mostly serious landscapes (bottom row). Visual inspection also indicates that the major difference of the artistic styles can be interpreted by visual features including color of pigments, intensity of ink, composition and stroke styles. As a result, it can be concluded that one visual feature alone is far from adequate to describe the variant artistic styles. Since color and texture features are widely reported in image processing communities, we simply adopt the features reported in [16] then focus on the analysis of composition and stroke features in the rest of this section to construct the integrated artistic style descriptor.

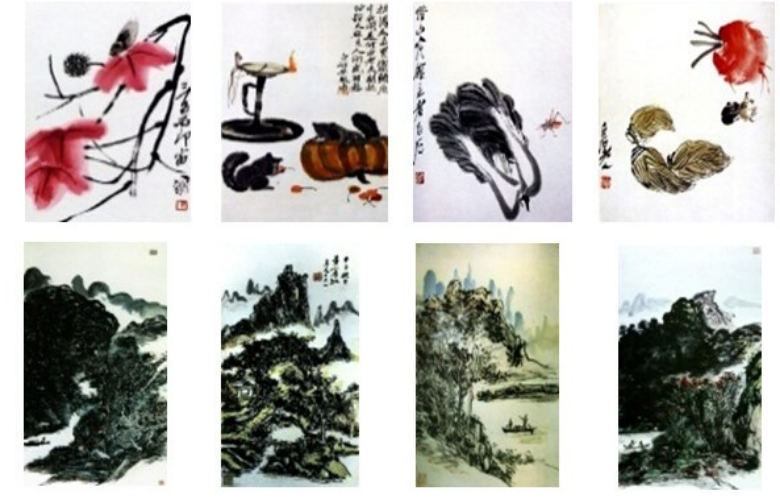

Fig. 1. Representative examples of traditional Chinese paintings with different styles. (First Row) Paintings of Qi Baishi and (Second Row) paintings of Huang Binhong

\subsection{Composition feature}

Composition inside traditional Chinese paintings serves as an important representation of artistic styles, which refers to the distribution of ink intensities in a given region, object, stroke or any contour-like edges etc. Some artists tend to paint in the neighborhood of central region, and some tend to fill in most of the regions. To extract such a feature and ensure it captures the draw styles of different Chinese artists, we divide a whole painting area into a number of sub-regions as shown in Fig. 2 . Then the Gray Level Co-occurrence Matrix (GLCM) [24] is using to measure the energy of sub-region. The GLCM is calculating how often a pixel with gray scale intensity value $i$ occurs adjacent to a pixel with the value $j$. Each element $(i, j)$ in GLCM specifies the number of times that the pixel with value $i$ occurred adjacent to a pixel with value $j$. For a given image $I$ of size $W \times H$, the element of a $G \times G$ gray-level co-occurrence matrix $M_{c o}$ for a displacement vector $\left(d_{x}, d_{y}\right)$ is defined as:

$$
M_{c o}=\sum_{x=1}^{W} \sum_{y=1}^{H}\left\{\begin{array}{c}
1, \text { if } I(x, y)=i \text { and } I\left(x+d_{x}, y+d_{y}\right)=j \\
0, \text { otherwise }
\end{array}\right\}
$$

Here the gray level $G$ is 8 , offset distance is 5 pixels and 4 directions of the offset (horizontal, vertical, $45^{\circ}$, and $135^{\circ}$ ). Then we evaluate each sub-region's energy.

$$
E=\sum_{i=1}^{G} \sum_{j=1}^{G} p(i, j)^{2}
$$

Here $p(i, j)$ is the normalized value of gray-level co-occurrence matrix $M_{c o}$. Finally the proposed composition feature CF is constructed by combining each sub-regions' energy together in their index order.

$\mathrm{CF}=\left\{\mathrm{E}_{1}, \mathrm{E}_{2}, \ldots, \mathrm{E}_{I}\right\}$

Where $E_{I}$ stands for the energy extracted from each sub-region.

Fig. 2 illustrates two examples of our proposed composition features, from which it can be seen that their composition features do illustrate significant differences with respect to their artistic styles. 


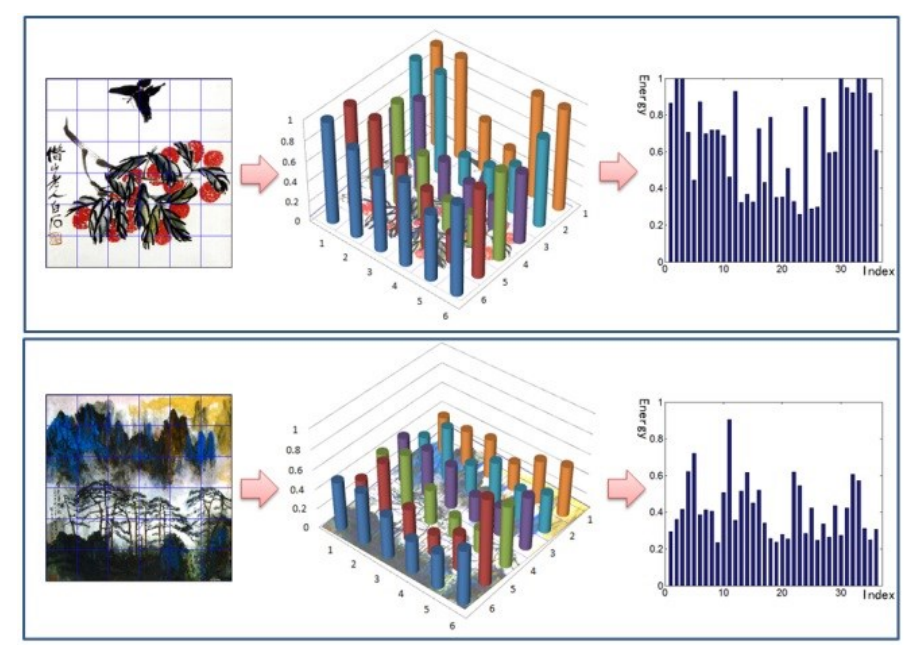

Fig. 2. The proposed composition features of two example paintings. Each painting is divided into $6 \times 6=36$ equal size sub-regions. (Top) A painting of Baishi's (1864-1957) and its high map of composition feature values. (Bottom) A painting of Haisu's (1896-1994) and its high map of composition feature values.

\subsection{Strokes Features of Chinese Painting}

Strokes are the fundamental units inside traditional Chinese paintings, not only reflecting the painters' brush techniques, but also describing the artists' styles, emotion and creative thoughts [25]. From visual point of view, while a stroke may have many different forms, shapes and directions inside the painting area, its physical description can still be characterized by its length, curvature and intensities. To this end, we carried out significant analysis and empirical studies upon the different styles of strokes. We firstly extract strokes of paintings by using the method proposed in [25] and then propose an integration of three features, including histogram of edge length, straightness and average stroke intensity, to formulate the stroke feature as defined below.

$\mathrm{SF}=\left\{\mathrm{E}_{\text {edgesize }}, \mathrm{S}_{\text {straightness }}, \mathrm{I}_{\text {intensity }}\right\}$

Where $\mathrm{E}_{\text {edgesize }}$ is the same as that reported in [16][18][20], and Fig. 3 shows two examples of such an edge size feature which indicates two different painting styles.

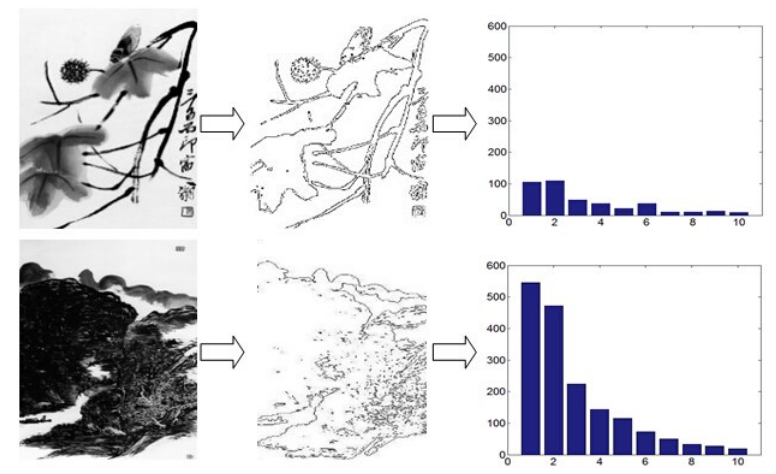

Fig. 3. Two paintings with different edge size statistical results. For each row, from left to right, they are Gray-level images, edge detection images and histogram of edge size statistical results.

While $\mathrm{S}_{\text {straightness }}$ is computed by using the absolute value of the linear correlation coefficient between the horizontal and vertical coordinates of the pixels located on the skeleton of the brushstroke. Suppose a brushstroke contains $\mathrm{N}$ pixels with coordinates $\left(\mathrm{x}_{i}, \mathrm{y}_{i}\right), \mathrm{i}=1, \ldots, \mathrm{N}$. The straightness is defined here.

$\mathrm{S}_{\text {straightness }}=\frac{N \sum_{i=1}^{N} x_{i} y_{i}-\sum_{i=1}^{N} x_{i} \sum_{i=1}^{N} y_{i}}{\sqrt{N \sum_{i=1}^{N} x_{i}^{2}-\left(\sum_{i=1}^{N} x_{i}\right)^{2}} \cdot \sqrt{N \sum_{i=1}^{N} y_{i}^{2}-\left(\sum_{i=1}^{N} y_{i}\right)^{2}}}$ 
Fig. 4 illustrates two examples with significant presence of straightness in their painting styles, which indicates that, even for the same content, different artists use different straightness to deliver their own artistic styles.
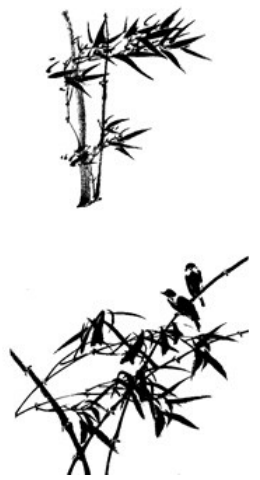
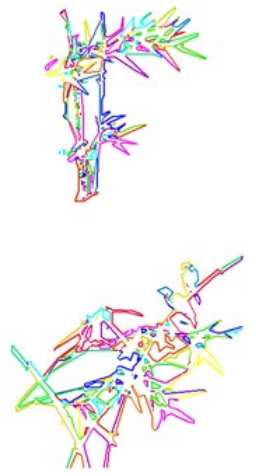

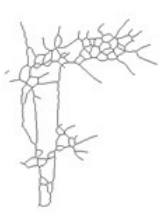

Straightness $=0.68$

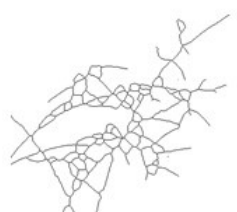

Straightness $=0.32$

Fig. 4. Illustration of Two Chinese paintings of bamboos with strong presence of stokes with different straightness. For each row, from left to right, they are Gray-level images filtered by thresholding, stroke detection color map and extracted skeleton of strokes. The top painting's average straightness of strokes is larger than the bottom one.

$\mathrm{I}_{\text {intensity }}$ is proposed and designed based on the fact that artists tend to use various strength and hence create different ink thickness to highlight their paintings. By selecting $N$ points on edges randomly and using each of these points as the center, we calculate the average pixel value of its neighboring $\mathrm{s} \times \mathrm{s}$ pixels. The average intensity of these patches is the stroke intensity, which is described as follows:

$\mathrm{I}_{\text {intensity }}=\frac{\sum_{i=1}^{N} \Sigma_{j=1}^{25}\left(P_{i, j} / s^{2}\right)}{N}$

Where $P_{i j}$ stands for the $j$ th pixel value in the $i$ th $5 \times 5$ sized rectangle.

Based on the above analysis of individual local and global visual features, an integrated artistic style descriptor can be initiated by combining all the features together, including the proposed composition feature, strokes feature, Ohta color histogram features (OF) and texture features (TF) which are | explicated in [18]. Let $P_{i}(i=1, \ldots, n)$ be the ith artist, such an integrated feature in describing its artistic styles can be constructed as follows:

$\mathrm{IF}\left(\mathrm{P}_{i}\right)=\left(\mathrm{CF}_{i}, \mathrm{SF}_{i}, \mathrm{OF}_{i}, \mathrm{TF}_{i}\right)$

However, in this classification problem, the discriminative power of each dimension is different according to different painting style. To automatically adaptive select most effective elements from the integrated feature for each artist, we proposed a Monte Carlo Convex Hull based dimension pruning and selection algorithm which is discussed in the next section.

\section{Monte Carlo Convex Hull Method for pruning towards optimization of the integrated descriptor}

In the integrated feature $\operatorname{IF}\left(\mathrm{P}_{i}\right)$, the contribution of each element is normally different, some elements make strong and positive contributions, and some make less strong or even negative contributions. If we do not exclude those less strong or negative elements the classification results will be affected and thus we regard these elements as noise in the process of describing the artistic style and completing their classifications.

To remove those less strong or negative elements from the integrated feature, we are facing the two problems, which can be described as: (i) to determine an upper bound for every individual artist as such that all the elements inside the integrated feature will make strong and positive contributions towards 
optimized classification; (ii) given the upper-bound, how to select those strong and positive dimensions included inside the feature set. To solve the first problem, we introduce a concept of "infusion factor: $s_{i} \in(0,1]$ ", inspired by following the chemical process with botanicals that release their active ingredients in water or alcohol, to describe the process of selecting and determining those active elements that make positive and strong contributions.

Each artist's training data can be regarded as a bundle of points in a high-dimensional space, which can be contained and described by a convex hull denoted as $\Omega($ Fig. 5). For the ith artist, as an example, some overlap of its convex hull $\Omega_{i}$ with other $\Omega_{j}$ which is calculated from $j t h$ artist's feature set is inevitable. Our observation and empirical studies also reveal that such overlapped regions are actually the main factors that cause the negative or less strong contributions for the integrated feature. Let $K$ be the number of the artists to be classified, we define discriminative power parameter $s_{i}$ of ith artist's paintings as follows:

$\mathrm{s}_{i}=1-\frac{\sum_{j=1, j \neq i}^{K} W_{i, j}}{K}$

$w_{i, j}=\frac{\mathrm{V}\left(\Omega_{i} \cap \Omega_{j}\right)}{\mathrm{V}\left(\Omega_{i}\right)}$

Where $w_{i, j}$ represents the proportion of the overlapped region between $\Omega_{i}$ and all other artist's convex hull $\Omega_{j}$, and $\mathrm{V}(\cdot)$ is the function that calculates the volume of the overlapped region.

To calculate $\mathrm{V}\left(\Omega_{i} \cap \Omega_{j}\right)$, one way is to calculate the shape and volume of $\Omega_{i}$ and $\Omega_{j}$ respectively, but its accurate derivation in a multidimensional space proves to be time consuming according to the definition of convex hull. To speed up the process and make the proposed algorithm practically useful without compromise on its performances, we propose to use clustering via k-means and the minimum bounding box algorithm [26] to estimate the overlapped region and hence determine the value of $w_{i, j}$. Let $F_{K}$ be the $k t h$ artist's integrated feature inside the whole training set, we firstly cluster $F_{K}$ into $\lambda$ sub-sets denoted as $F_{K \lambda}$, we then combine every $F_{K \lambda}$ 's minimum bounding box together as an approximation of $\Omega_{k}$, which can be described as follows:

$\Omega_{k} \approx B\left(F_{k 1}\right) B\left(F_{K 2}\right) \cup \ldots \cup B\left(F_{k \lambda}\right)$

Where $B(\cdot)$ is the minimum bounding box algorithm which has been extended to multi-dimensional. About the cluster number $\lambda$, it affects how the bounding box fits the boundary of feature set. It results in the loss of accuracy if the cluster number is too small. On the contrary the accuracy is high but it causes the increasing of computational complexity. According to our empirical | experiment, the value of parameter $\lambda$ is 3 in this paper. Fig. 5 shows a demonstration for the case with two-dimensions.

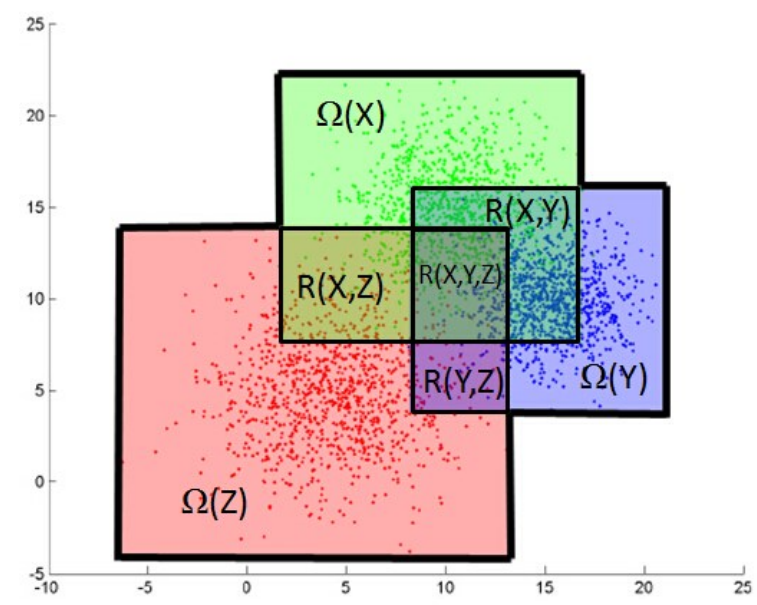

Fig. 5. Illustration of how $\operatorname{overlap}\left(\Omega_{i}, \Omega_{j}\right)$ is calculated. 
To calculate the specific value of $w_{i, j}$, we adopt the Monte Carlo algorithm to obtain the estimated results.

As Fig. 5 shows, the feature convex hull $\Omega(\mathrm{X}), \Omega(\mathrm{Y})$ and $\Omega(\mathrm{Z})$ are overlapped with each other. The overlapped regions $\mathrm{R}(\mathrm{X}, \mathrm{Y}), \mathrm{R}(\mathrm{X}, \mathrm{Z}), \mathrm{R}(\mathrm{Y}, \mathrm{Z})$ and $\mathrm{R}(\mathrm{X}, \mathrm{Y}, \mathrm{Z})$ contain the most correlate features and indicate the noise level of $\Omega_{i}$. Firstly, we randomly generate $\alpha$ points in the space of $\Omega_{i}$, and then calculate how many points fall into other convex hull $\Omega_{j}$ to estimate the overlap between $\Omega_{i}$ and $\Omega_{j}$. Let the number of these points in overlapped regions be $\mathrm{y}(\mathrm{i} \mid \mathrm{j})$, the value of $w_{i, j}$ can be estimated as:

$w_{i, j} \approx \frac{y(i \mid j)}{\alpha}$

Then the discriminative power parameter $\mathrm{s}_{i}$ of $i$ th artist's feature set can be calculated according to Eq. (8) and (9). As a result, let $\mathrm{N}_{I F}$ be the total number of elements inside the integrated feature of IF in formulation (7), the upper bound of selected feature is denoted as $\mathrm{N}_{I F}^{\prime}$ for the ith artist, can be calculated as follows:

$\mathrm{N}_{I F}^{\prime}=\left\lceil N_{I F} \times s_{i}\right\rceil$ and $0<\mathrm{s}_{i} \leq 1$

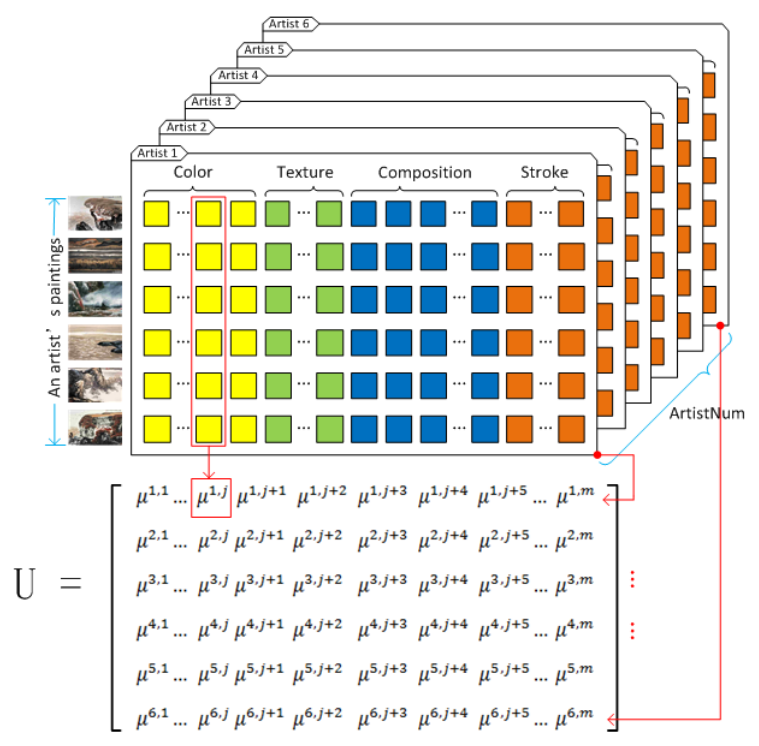

Fig. 6. Illustration of mapping training dataset to the matrix $U$.

For the convenience of describing our proposal to resolve the second problem, the entire training I dataset for all the artists can be summarized in a multi-dimensional space as shown in Fig. 6. As seen, each layer represents all the integrated features extracted from the training paintings for one artist, inside which each column lists one element of the integrated feature across all the training paintings as | illustrated on the left of Fig. 6. To select those elements which make positive and strong contributions to the classification process, we extract a matrix of means $U$ for all elements inside the integrated | features across all artists as shown at the bottom of Fig. 6.

To examine the strength of each individual element inside the $m$-dimensional integrated feature with respect to all other elements, we further extract $\sigma_{i, j}^{2}$, the variance of $j$ th column inside matrix $\mathrm{U}$ for the $i t h$ artist, and $\hat{\sigma}_{i, j}^{2}$, the average difference between $\mu^{\mathrm{i}, \mathrm{j}}$ and all other means in the same column of the matrix $U$, which is calculated as follows:

$\hat{\sigma}_{i, j}^{2}=\frac{\sum_{i^{\prime}=1, i^{\prime} \neq i}^{\operatorname{ArtistNum}}\left(\mu^{\mathrm{i}^{\prime}, \mathrm{j}}-\mu^{\mathrm{i}, \mathrm{j}}\right)^{2}}{\operatorname{ArtistNum}}$

As a matter of fact, $\sigma_{i, j}$ measures the level of differences among all the mean values for the $j$ th element inside the integrated feature across all artists' training set, and hence a smaller value indicates that it is less likely to be disturbed by others and a higher rank should be allocated. In contrast, $\hat{\sigma}_{i, j}^{2}$ 
measures the average difference of the $j$ th element inside the integrated feature across all artists, and the larger the value is, the less likely this element will cause large overlap across different artists. Therefore, $\hat{\sigma}_{i, j}^{2}$ indicates the discriminating power of the $j t h$ element inside the integrated feature, and we introduce a rank value $\xi_{i, j}$ to select those strong and positive elements inside the integrated feature. $\xi_{i, j}$ is defined as given below:

$\xi_{i, j}=\frac{\sigma_{i, j}}{\widehat{\sigma}_{i, j}}$

The smaller the value of $\xi_{\mathrm{i}, \mathrm{j}}$, the higher the corresponding element is ranked and hence more likely to be selected for the pruned artistic style descriptor.

An example of the proposed feature pruning processing is shown below. Given the ith artistic feature descriptor $\mathrm{F}_{i}=\left\{f_{i j} \mid j=1, \ldots, M\right\}$, then we rank the $\mathrm{f}_{i j}$ 's position according the corresponding value of $\xi_{\mathrm{i}, \mathrm{j}}$ in ascending order, denoted as $\mathrm{F}_{i}{ }^{\prime}=\left\{f_{i j}{ }^{\prime} \mid j=1, \ldots, M\right\}$. Finally according the Eq. (11) we calculate $\mathrm{n}_{i}$ which means the number of feature's elements should be kept, so the feature pruning result is $\overline{\mathrm{F}}_{i}^{\prime}=\left\{f_{i j}^{\prime} \mid j=1, \ldots, n\right\}$.

Finally the integrated feature elements are sorted and pruned by using the proposed $\mathrm{MCCH}$ method. $\mathrm{IF}^{\prime}\left(\mathrm{P}_{i}\right)=\operatorname{MCCH}\left(\mathrm{CF}_{i}, \mathrm{SF}_{i}, \mathrm{OF}_{i}, \mathrm{TF}_{i}\right)$

To construct the MCCH-SVM classifier model, the MCCH pruned features are pour into a SVM classifier [27] whose source code can be found here (http://www.csie.ntu.edu.tw/ cjlin/). Fig. 7 shows the MCCH-SVM classifier we proposed in this paper.

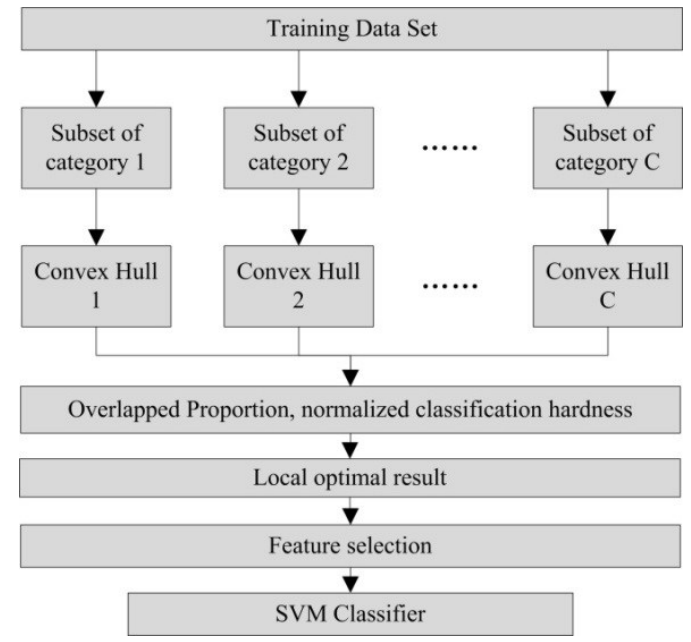

Fig. 7. The proposed architecture of MCCH-SVM model.

\section{Experimental Results and Evaluation Analysis}

To evaluate the proposed algorithm, we have set up a test database of 180 traditional Chinese paintings with 6 famous traditional Chinese artists. The database is not very large because it is not an easy task to collect high quality digital paintings, especially those from famous artists. We applied the proposed MCCH-SVM for the completed experimental evaluations via automated classification of all traditional Chinese paintings corresponding to their authored artists. To validate the efficacy of the proposed model, a 5-fold cross-validation is used in our experiments. For the collected painting samples, $80 \%$ of the images from each of the six artists are employed for training, and the rest $20 \%$ are used for testing to evaluate the classification performance of the proposed algorithm.

For the convenience of benchmarking, we also tested three cases of the classification, which 
are highlighted as follows:

Case-1: classification among two artists with Dai and Huang Z;

Case-2: classification among four artists with Dai, Huang Z, Qi and Shi;

Case-3: classification among all six artists with Dai, Huang Z, Qi, Shi, Huang BH, Liu.

For performance assessment, the proposed method has been benchmarked with four state-of-the-art approaches, which include MHMM with its own feature sets and classification method, Convolutional Neural Networks (CNN) method [28], Multi-kernel Learning method [29] and sparse lasso algorithm [30] with features extracted using the approach introduced in [18]. As the dataset in [16] is not publicly available, all these approaches are tested on the dataset we collected. Table 1 summarizes the experimental results for all the three cases.

Table 1

Summary of experimental results in terms of precision and recall rates.

Case1

\begin{tabular}{|c|c|c|c|c|c|c|c|c|c|c|c|}
\hline \multirow{2}{*}{$\begin{array}{l}\text { Precision }(\mathrm{P}) \\
\& \operatorname{Recall}(\mathrm{R})\end{array}$} & \multicolumn{2}{|c|}{ Proposed } & \multicolumn{2}{|c|}{ MHMM } & \multicolumn{2}{|l|}{$\mathrm{CNN}$} & \multicolumn{2}{|l|}{ MKL } & \multicolumn{3}{|c|}{ Sparse lasso } \\
\hline & $\mathrm{P}$ & $\mathrm{R}$ & $\mathrm{P}$ & $\mathrm{R}$ & $\mathrm{P}$ & $\mathrm{R}$ & $P$ & & $\mathrm{P}$ & \multicolumn{2}{|c|}{$\mathrm{R}$} \\
\hline Dai & 0.98 & 0.97 & 0.7 & 0.72 & 0.92 & $0.92 \quad 0$. & $0.97 \quad 0$ & .920 & 0.95 & \multicolumn{2}{|c|}{0.93} \\
\hline Huang Z & 0.96 & 0.99 & 0.92 & 0.9 & 0.96 & $0.96 \quad 0$. & $0.91 \quad 0$ & .960 & 0.94 & \multicolumn{2}{|c|}{0.89} \\
\hline Average & 0.97 & 0.95 & 0.81 & 0.81 & 0.94 & $0.94 \quad 0$. & $0.94 \quad 0$ & .940 & 0.95 & \multicolumn{2}{|c|}{0.91} \\
\hline \multicolumn{12}{|c|}{ Case2 } \\
\hline Precision $(\mathrm{P})$ & \multicolumn{2}{|c|}{ Proposed } & \multicolumn{2}{|c|}{ MHMM } & \multicolumn{2}{|l|}{$\mathrm{CNN}$} & \multicolumn{2}{|l|}{ MKL } & \multicolumn{3}{|c|}{ Sparse Lasso } \\
\hline$\&$ Recall(R) & $\mathrm{P}$ & $\mathrm{R}$ & $\mathrm{P}$ & $\mathrm{R}$ & $\mathrm{P}$ & $\mathrm{R}$ & $\mathrm{P}$ & $\mathrm{R}$ & $\mathrm{P}$ & \multicolumn{2}{|c|}{$\mathrm{R}$} \\
\hline Dai & 0.98 & 0.97 & 0.64 & 0.62 & 0.91 & 0.9 & 0.92 & 0.9 & 0.94 & \multicolumn{2}{|c|}{0.91} \\
\hline Huang Z & 0.9 & 0.9 & 0.72 & 0.7 & 0.82 & 0.82 & 0.9 & 0.85 & 0.9 & \multicolumn{2}{|c|}{0.88} \\
\hline Shi & 1 & 1 & 0.9 & 1 & 0.83 & 0.98 & 1 & 1 & 0.92 & \multicolumn{2}{|c|}{0.9} \\
\hline Qi & 0.98 & 0.82 & 0.7 & 0.7 & 0.86 & 0.6 & 0.66 & 0.9 & 0.84 & \multicolumn{2}{|c|}{0.85} \\
\hline Average & 0.97 & 0.92 & 0.74 & 0.76 & 0.86 & 0.83 & 0.87 & 0.91 & 0.9 & & 89 \\
\hline & & & & & Case3 & & & & & & \\
\hline Precision $(\mathrm{P})$ & Propos & & MHMM & & $\mathrm{CNN}$ & & MKL & & & Sparse & asso \\
\hline \& Recall(R) & $\mathrm{P}$ & $\mathrm{R}$ & $\mathrm{P}$ & $\mathrm{R}$ & $\mathrm{P}$ & $\mathrm{R}$ & $\mathrm{P}$ & $\mathrm{R}$ & & $\mathrm{P}$ & $\mathrm{R}$ \\
\hline Dai & 0.98 & 0.97 & 0.56 & 0.62 & 0.77 & 0.88 & $\begin{array}{ll}8 & 0.91\end{array}$ & $0.8 \mathrm{~s}$ & & 0.93 & 0.91 \\
\hline Huang Z & 0.9 & 0.9 & 0.66 & 0.68 & 0.8 & 0.8 & 0.9 & 0.77 & & 0.9 & 0.85 \\
\hline Shi & 1 & 1 & 0.7 & 0.74 & 0.8 & 0.84 & $4 \quad 1$ & 1 & & 0.8 & 0.9 \\
\hline Qi & 0.96 & 0.8 & 0.53 & 0.62 & 0.84 & 0.6 & 0.64 & $4 \quad 0.8$ & & 0.82 & 0.75 \\
\hline Huang BH & 0.88 & 0.96 & 0.8 & 0.7 & 0.83 & 0.88 & $\begin{array}{ll}8 & 0.8\end{array}$ & 0.9 & & 0.92 & 0.88 \\
\hline Liu & 0.98 & 0.92 & 0.65 & 0.5 & 0.81 & 0.8 & 0.7 & 0.9 & & 0.86 & 0.87 \\
\hline Average & 0.95 & 0.93 & 0.65 & 0.64 & 0.81 & 0.8 & 0.83 & 30.88 & & 0.88 & 0.86 \\
\hline
\end{tabular}

Comparing with the MHMM method and CNN methods, the proposed delivers considerably better results on average of precision and recall in all the three cases. As seen, when the number of artists is increased, the classification results achieved by the proposed actually reduces slower than that of the other two benchmarks.

To test the robustness of the proposed artistic style descriptor, we revise the classification experiment as such that one more additional class, denoted as "unknown", is added to represent those paintings that do not belong to any of these six artists. As a result, the training set is revised by randomly selecting some traditional Chinese paintings authored by those Chinese artists other than the 
included six, yet their content and style are also selected to be close to those existing authored paintings. | With such more strict settings, the experimental results are summarized in Table 2, from which it can be seen that the precision rate of Qi Baishi is reduced due to the fact that a lot of the paintings inside the additional class are similar to Qi's. Such selection is arranged on purpose in order to test the robustness of the proposed style descriptor. Some examples of the paintings that are incorrectly | classified as Qi Baishi are illustrated in Fig. 8. For comparison, four real Qi Baishi's paintings are shown in the top row, it can be perceived that these wrong assigned paintings show very similar painting style of real Qi Baishi's painting style. All the results indicate that the proposed artistic style descriptor does have the capability in distinguishing the paintings with different drawing styles. For some paintings with similar artistic styles, however, further work is still needed to further improve the classification accuracy.

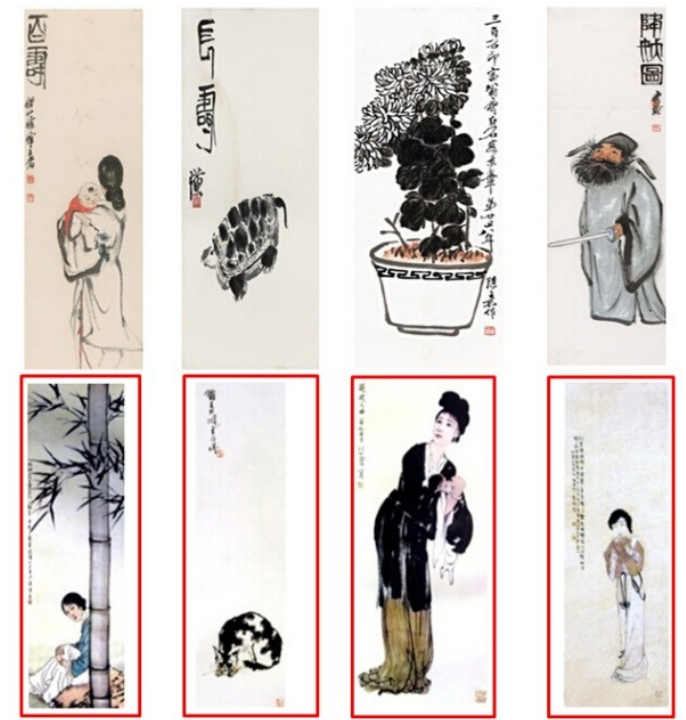

Fig. 8. Comparison of four real paintings from Qi Baishi (top) and four samples from unknown artists being incorrectly assigned to Qi Baishi (bottom).

Table 2

Experimental results for testing the robustness of the proposed method

\begin{tabular}{lll}
\hline & Precision & Recall \\
\hline Dai & 0.96 & 0.92 \\
Huang Z & 0.9 & 0.9 \\
Shi & 0.95 & 0.95 \\
Qi & 0.93 & 0.82 \\
Huang BH & 0.85 & 0.94 \\
Liu & 0.92 & 0.91 \\
Unknown & 0.71 & 0.5 \\
Average & 0.889 & 0.849 \\
\hline
\end{tabular}

To further benchmark the proposed feature dimension pruning and selecting scheme, we further applied the well-known sequential forward floating selection (SFFS)with cross validation (CV) scheme | to process the integrated feature as given in Eq. (6), the proposed with or without the feature selection method. Then we repeat some of the experiments in case-3 as described above in Table 1. The | corresponding experimental results are shown in Table 3, which support that the proposed feature selection method play important role in the proposed method and outperforms the SFFS-based cross validation schema. 
Table 3

Comparative experimental results with SFFS-based cross validation

\begin{tabular}{lll}
\hline & Average Precision & Average Recall \\
\hline SFFS\&CV & 0.78 & 0.73 \\
$\begin{array}{l}\text { Proposed without } \\
\text { feature selection }\end{array}$ & 0.88 & 0.86 \\
Proposed & 0.95 & 0.93 \\
\hline
\end{tabular}

\section{Conclusion}

In this paper, we have described an integrated artistic style descriptor for characterizing the traditional Chinese paintings, where a new concept of infusion factor is introduced to facilitate a self-adaptive pruning scheme for processing the integrated feature. Via analyzing the visual cues of Chinese paintings, the pruned artistic style descriptor includes a number of low-level features that are proved to play crucial roles in representing and reflecting relevant artistic styles. These include color, texture, composition and strokes. Considering the fact that simple integration of all these features fail to maximize their discriminating power, detailed analysis and empirical studies are carried out and hence a self-adaptive pruning scheme has been proposed to capture the correlations and the synergy across all different features, dimensional elements and their roles in terms of their performances as well as contributions in the training process. Experimental results reveal that the proposed algorithm outperforms the existing representative benchmarks, including MHMM and Convolutional Neural Networks method.

In comparison with the existing techniques reported in the literature, the contributions of our proposed algorithm described in this paper can be highlighted as: (i) analysis of visual cues inside traditional Chinese paintings and hence design of new visual features to describe the traditional artistic styles; (ii) introduction of a new Monte Carlo Convex Hull based method for self-adaptive pruning scheme to optimize the structure of the integrated artistic style descriptor. Experiments support that the proposed MCCH-SVM classifier not only outperforms the existing representative benchmark but also achieves certain level of robustness as evidenced by the experimental results illustrated in Table 2 and Table 3.

\section{Acknowledgments}

The authors wish to acknowledge the support from the National Natural Science Foundation, China, under the grants [61003201], [61211130125], [61202165] and [61272266].

\section{References}

[1] A. Goldberg, X. Zhu, A. Singh, Z. Xu, R. Nowak, Multi-manifold semi-supervised learning, in: Proceedings of 12th International Conference on Artificial Intelligence and Statistics, 2009, pp.169-176.

[2] C. Chang, C. Lin, LIBSVM: a library for support vector machines, ACM Transactions on Intelligent Systems and Technology 2 (27) (2011) 1-27.

[3] F.F. Li, P. Perona, A bayesian hierarchical model for learning natural scene categories, in: Proc. of the 2005 IEEE Computer Society Conf. on Computer Vision and Pattern Recognition, 2005, pp. 524-531.

[4] K. Grauman, T. Darrell, The pyramid match kernel: discriminative classification with sets of image features, in: Proceedings of the International Conference on Computer Vision, 2005, pp.1458-1465.

[5] S. Lazebnik, C. Schmid, J. Ponce, Beyond bags of features: spatial pyramid matching for recognizing natural scene categories, in: Proceedings of the Computer Vision and Pattern Recognition, 2006, pp. 2169-2178.

[6] Z. Wu, Q. Ke, M. Isard, J. Sun, Bundling features for large scale partial-duplicate web image search, in: Proceedings of the Computer Vision and Pattern Recognition, 2009, pp.25-32.

[7] X. Wang, X. Bai, W. Liu, L. Latecki, Feature context for image classification and object detection, in: Proceedings of the Computer Vision and Pattern Recognition, 2011, pp.961-968. 
[8] C. Zhang, J. Liu, Q. Tian, Y. Han, H. Lu, S. Ma, A boosting sparsity-constrained bilinear model for object recognition, IEEE Multimedia 19 (2) (2012) 58-68.

[9] B. Yao, A. Khosla, F.F. Li, Classifying actions and measuring action similarity by modeling the mutual context of objects and human poses, in: Proceedings of the International Conference on Machine Learning, 2009.

[10] Y. Lee, K. Grauman, Object-graphs for context-aware category discovery, in: Proceedings of the Computer Vision and Pattern Recognition, 2010, pp.1-8.

[11] B.D. Liu, Y.X. Wang, Y.J. Zhang, B. Shen, Learning dictionary on manifolds for image classification, Pattern Recognition 46 (7) (2013) 1879-1890.

[12] J. Yang, K. Yu, Y. Gong, T. Huang, Linear spatial pyramid matching using sparse coding for image classification, in: IEEE Conference on Computer Vision and Pattern Recognition, 2009, pp.1794-1801.

[13] T. Serre, L. Wolf, T. Poggio, Object recognition with features inspired by visual cortex, in: IEEE Conference on Computer Vision and Pattern Recognition, 2005, pp.994-1000.

[14] J. Wang, J. Yang, K. Yu, F. Lv, T. Huang, Y. Gong, Locality-constrained linear coding for image classification, in: IEEE Conference on Computer Vision and Pattern Recognition, 2010, pp.3360-3367.

[15] C. Zhang, S. Wang, Q. Huang, Image classification using spatial pyramid robust sparse coding, Pattern Recognition Letters 34 (2013) 1046-1052.

[16] J. Li, J.Z. Wang, Studying digital imagery of ancient paintings by mixtures of stochastic models, IEEE Trans. Image Process. 13 (3) (2004) 340-353.

[17] I.E. Berezhnoy, E.O. Postma, J. van den Herik, Computerized visual analysis of paintings, in: Proceeding of International Conference on Association for History and Computing, 2005, pp. 28-32.

[18] S. Jiang, Q. Huang, Q. Ye, W. Gao, An effective method to detect and categorize digitized traditional Chinese paintings, Pattern Recognition Lett. 27 (7) (2006) 734-746.

[19] J. Yang, Y. Jiang, A.G. Hauptmann, C. Ngo, Evaluating bag-of-visual-words representations in scene classification, in: Proc. Multimedia Information Retrieval, 2007, pp.197-206.

[20] C.R. Johnson, J.E. Hendriks, I. Berezhnoy, E. Brevdo, S. Hughes, I. Daubechies, J. Li, E. Postma, J.Z. Wang, Image processing for artist identification: brushwork in the paintings of Vincent Van Gogh, IEEE Signal Processing Magazine 25 (4) (2008) 37-48.

[21] J. Shen, Stochastic modeling western paintings for effective classification, Pattern Recognition 42 (2) 2009 293-301.

[22] J. Qin, N. Yung, Scene categorization via contextual visual words, Pattern Recognition 43 (5) (2010) 1874-1888

[23] J. Uijlings, A. Smeulders, R. Scha, Real-time visual concept classification, IEEE Transactions on multimedia 12 (7) (2010) 665-681.

[24] R.M. Haralick, K. Shanmugam, I. Dinstein, Textural features for image classification, IEEE Transactions on Systems, Man, and Cybernetics 3 (6) (1973) 610-621.

[25] J. Li, L. Yao, E. Hendriks, J.Z. Wang, Rhythmic brushstrokes distinguish van Gogh from his contemporaries: findings via automated brushstroke extraction, IEEE Transactions on Pattern Analysis and Machine Intelligence 34 (6) (2012) 1159-1176.

[26]G. Barequet, S. Har-Peled, Efficiently approximating the minimum-volume bounding box of a point set in three dimensions, Journal of Algorithms 38 (1) (2001) 91-109.

[27] C. Nello, S.T. John, An Introduction to Support Vector Machines and other kernel-based learning methods, Cambridge University Press, 2000, ISBN 0-521-78019-5.

[28] X.X. Niu, C.Y. Ching, A novel hybrid CNN-SVM classifier for recognizing handwritten digits, Pattern Recognition 45 (4) (2012) 1318-1325.

[29]Serhat S. Bucak, Rong Jin, and Anil K. Jain, Multiple Kernel Learning for Visual Object Recognition: A Review, IEEE Transactions on Pattern Analysis and Machine Intelligence, 36(7), 1354-1369, 2013.

[30]Jun Liu, Shuiwang Ji, and Jieping Ye. SLEP: Sparse Learning with Efficient Projections[OL][2012-09-24] http://www.public.asu.edu/ jye02/Software/SLEP/ 